\title{
Possible Therapeutic Role of Bone Marrow-Derived Mesenchymal Stem Cells in a Rat Model of Hepatocellular Carcinoma
}

\author{
Fathy M. El-taweel ${ }^{1}$, Sara A. El-helf ${ }^{1}$, Marihan A. Helal ${ }^{1}$ and Hatem A. El-mezayn ${ }^{* 2}$ \\ ${ }^{1}$ Chemistry Department, Faculty of Science, Damietta University, Damietta, Egypt. \\ ${ }^{2}$ Chemistry Department, Helwan University, Cairo, Egypt.
}

Received: 5 May 2021 /Accepted: 8 May 2021

*Corresponding author's E-mail: hatem_mezayezayen@science.helwan.edu.eg

\begin{abstract}
Hepatocellular carcinoma (HCC) is one of the most common types of cancer and its high mortality and short survival time causes a serious worldwide health burden. Chemotherapy as 5-Flourouracil (5-FU) is used as the first-choice treatment for HCC, however it is associated with resistance, low efficacy and has side effects. Consequently, the administration of mesenchymal stem cells (MSCs) as a novel therapy for HCC holds great promise. MSCs can differentiate into hepatocytes, reduce liver inflammation, promote hepatic regeneration and secrete protective cytokines. This is the first study that sheds new light on the role of MSCs alone or together with 5-FU in HCC. In this study, Bone marrow derived-MSCs were prepared and HCC model was induced via treating rats with Ndiethylnitrosamine (DEN). The effect of treatments was evaluated by biochemical, tumor markers, macroscopical, and histopathological analysis. Also, cell cycle, apoptosis assay, KI67 and CD95 were performed by Flowcytometry. The administration of MSCs together with 5-FU yields a decrease in the liver enzymes with down-regulation of tumor markers as well as improvement of histopathological picture. In addition, such treatment decreased cell proliferation that associated with the down-regulation of KI67 expression. Furthermore, MSCs did not mediate the apoptosis pathway together with reduction of CD95 expression and cell cycle arrest at G0/G1 phase. In conclusion, MSCs exerted antitumor effects by inhibiting proliferation, modulating the cell cycle in G0/G1 phase and improved the toxic effect of 5FU via improvement of liver enzymes and decreased tumor markers.
\end{abstract}

Keywords: .

\section{Introduction}

Hepatocellular carcinoma (HCC), the crucial malignant tumor of the liver, has become the third foremost cause of cancer death worldwide, with more than 500,000 new cases diagnosed each year (Hemming, Berumen et al. 2016). HCC development results from an imbalance between excessive cell growth and apoptosis, which is mostly regulated by P53, a tumor suppressor gene (Serhal, Saliba et al. 2019). Moreover, HCC occurs mainly in patients with fundamental chronic liver disease and cirrhosis with a variety of molecular causes, local expansion, intrahepatic spread, and ultimately distant metastatic progression (Greten 2020). In recent years, although the 
level of screening, diagnosis and treatment of liver cancer has increased, the incidence and mortality of cancer have been increasing (Moris, $\mathrm{Lu}$ et al. 2017). Furthermore, intrahepatic metastasis of primary liver cancer can lead to tumor recurrence, making the treatment of liver cancer very difficult. Therefore, it is urgent to develop new methods to control the growth and metastasis of liver cancer clinically (Liu, Li et al. 2020).

Also, the ineffective treatment and poor prognosis are common for lack of knowledge about mechanism of hepatocarcinogenesis. Despite the existence of many cancer treatments, including curative surgical resection, chemotherapy, radiation therapy, liver transplantation and radiofrequency ablation, these therapeutic effects are still not particularly desirable (Stoltz, Gagnière et al. 2014). Systemic chemotherapy is the main treatment for HCC. However, traditional chemotherapy treatments provide little efficacy in patients with advanced HCC and often provide no benefit to survival rate (Daher, Massarwa et al. 2018).

Among the chemotherapeutic drugs used in HCC, 5-Fluorouracil (5-FU), a pyrimidine based analog, was considered as one of the firstline chemotherapy drugs for the treatment of malignant tumors including liver, breast and other digestive system tumors (Liu, Zhao et al. 2020). Indeed, 5-FU was an important antimetabolite and cell cycle-targeting drug that proved to be beneficial in treating advanced HCC (Qin, Bai et al. 2013). However, the routine dose of 5-FU is accompanied by serious side effects, including leukopenia, nausea, emesis, and skin reactions (Hu, Lv et al. 2016). In addition to its inevitable toxicity to normal cells and multidrug resistance caused by longterm use (Garg, Lincz et al. 2012). It has been established that, the combination therapy with 5-FU improved the response rate in advanced HCC patients (Damdinsuren, Nagano et al. 2007, Sho, Nakanishi et al. 2017). Therefore, there is an urgent need to improve the sensitivity of HCC to chemotherapy, and to reduce drug-dosage and then reduce their side effects.

Mesenchymal stem cells (MSCs) have become an attractive matter of study for treatment of HCCs (Wu, Zhang et al. 2016). MSCs are adult, multipotent, nonhematopoietic cells that have auto-renewing capacity and a multilineage potential, which can produce a variety of differentiated cell/tissue lineages, including cartilage, bone, adipose tissue, tendons, and ligaments and have low tumorigenicity (Godwin, Young et al. 2012). MSCs also exhibit comparatively low immunogenicity and do not stimulate lymphocyte proliferation, thus avoiding immune rejection (Li, Ren et al. 2019). These characteristics of MSCs construct them very suitable for cell therapy. The most attractive feature of MSCs is that they can specifically migrate to the tumor site, which has led to increased interest in using MSCs as carriers to deliver anti-tumor drugs or genes for cancer treatment (Babajani, Soltani et al. 2020). Subsequently, it has been proposed as a promising tool for the reduction of tumor growth and metastasis. However, whether MSCs suppress or promote HCC development is still controversial. Several studies indicated that MSCs control liver cancer growth (Yulyana, Ho et al. 2015), whereas others showed that MSCs promoted tumor progression and metastasis in animal models (Hernanda, Pedroza-Gonzalez et al. 2013). The present study is performed to throw more lights on the effect of MSCs alone or combined therapy with 5-FU on HCC. To the best of our knowledge, this is the first study that evaluates the combination effectiveness between these two treatments in albino rats' model of HCC.

\section{Material and Methods}

\section{Chemicals and reagents}

Dulbecco's Modified Eagle's medium (DMEM), ethylenediaminetetraacetic acid (EDTA) and penicillin-streptomycin were purchased from, lonza, Belgium. Fetal bovine serum (FBS) was purchased from seralab, Brazil. Trypan blue was purchased from lonza, USA. Diethylnitrosamine (DEN) purchased from Sigma-Aldrich (St. Louis, MO, USA). $5 \mathrm{FU}$ was purchased from EIMC United Pharmaceuticals, Cairo, Egypt. propidium iodide (PI) was purchased from Invitrogen; Thermo Fisher Scientific, Inc.

\section{Preparation of bone marrow derived MSCs}

Bone marrow derived-MSCs were prepared at Nile center for experimental research, Mansoura, Egypt. The method used 
was a modification of that of Abdel aziz et al.(Abdel aziz, El Asmar et al. 2011). Bone marrow was collected from the long bones of male rats, and washed with DMEM supplemented with $10 \%$ FBS and $1 \%$ penicillin-streptomycin. Unattached cells were removed daily, and adherent cells were kept in the incubator at $37^{\circ} \mathrm{C}$ in $5 \%$ humidified $\mathrm{CO}_{2}$ for $10-15$ days as primary culture. When the cells reached $80-90 \%$ confluence, they were washed twice with phosphate buffer saline (PBS), and trypsinized with $0.25 \%$ trypsin in $1 \mathrm{mM}$ EDTA for $5 \mathrm{~min}$ at $37^{\circ} \mathrm{C}$. Cells were then centrifuged and resuspended in $50 \mathrm{~cm}^{2}$ culture flasks (falcon), and incubated (first passage cultures). Cell cultures were routinely assessed with the use of an inverted microscope (Olympus CKX41, USA), and cell viability was determined by means of trypan blue $0.4 \%$ staining. MSCs in culture were characterized by their adhesiveness and spindle shape, which was detected by means of an inverted microscope and through determination of surface markers of bone marrow MSCs. The latter was performed through evaluation of the positive expression of nonhematopoietic origin [by use of the monoclonal antibodies that recognize an epitope on endoglin (CD29, CD44)] and the negative expression of hematopoietic markers, such as CD34, which were analyzed by means of flow cytometer according to Calabro et al.(Calabrò, Fonsatti et al. 2003).

\section{Animals}

Adult male albino rats with an average body weight of 120-160 g purchased from Theodore Bilharz Institute, Giza, Egypt were used. Rats were fed with the standard rat diet and water ad libitum. They were acclimatized to the experimental room having a temperature of $23 \pm 2{ }^{\circ} \mathrm{C}$, controlled humidity conditions, and $12 / 12 \mathrm{~h}$ light and dark cycle according to the criteria outlined by the National Institutes of Health (NIH) (Council 2010).

\section{Preparation of HCC Model}

DEN is a strong hepatocarcinogenic nitrosamine was used for induction of HCC. DEN was administered intragastrically to the rats at the dose of $10 \mathrm{mg} / \mathrm{kg} 5$ times a week during 2 months (Karimov, Inoyatova et al. 2003)

\section{Experimental design}

Animals were randomly divided into five groups; each group was comprised of eight animals except group 2 which was comprised of ten animals ( 2 rats were used for the confirmation of the induction of HCC at the end of 9th week of the experiment by the histopathological examination of rat livers).

Group (1): control group of healthy male rats as negative control group.

Group (2): HCC group in which rats were injected intragastrically with $10 \mathrm{mg} / \mathrm{kg}$ DEN 5 times a week during 2 months.

Group (3): 5FU treated group in which rats with HCC was treated with 5FU intraperitoneally as $75 \mathrm{mg} / \mathrm{kg}$ body weight, diluted with saline, once per week for two consecutive weeks (Winocur, Vardy et al. 2006).

Group (4): BM-MSCs treated group in which rats with $\mathrm{HCC}$ was treated with a single dose of BM derived MSCs $\left(3 \times 10^{6}\right)$ cells per rat intravenously (Abdel aziz, El Asmar et al. 2011).

Group (5): 5FU + BM-MSCs treated group in which rats with $\mathrm{HCC}$ was treated with $5 \mathrm{FU}$ intraperitoneally as $37.5 \mathrm{mg} / \mathrm{kg}$ body weight, diluted with saline, once per week for two consecutive weeks, followed by a single dose of $\mathrm{BM}$ derived MSCs $\left(3 \times 10^{6}\right)$ cells per rat intravenously.

At the end of the experiment, the blood samples and liver tissues were collected from animals for biochemical, flow cytometric and histological assays.

\section{Histopathological analysis}

A sample of liver obtained after decapitation was washed in saline and fixed in $10 \%$ formalin for the routine haematoxylin and eosin (H\&E) staining technique and histopathological examinations. Fixed liver tissue samples were dehydrated by passing in ascending series of alcohol then cleaned with xylene and embedded in paraffin wax. Sections of the tissues 5-6 $\mu \mathrm{m}$ thickness were prepared 
by using a rotary microtone and stained with hematoxylin and eosin (H\&E) dye. The pathologist evaluating liver sections was unaware of the treatment the rat had received.

\section{Biochemical evaluation}

Serum samples obtained from the rats were analyzed for alanine aminotransferase (ALT) and aspartate aminotransferase (AST) using commercial kits (BioSystems S.A., Barcelona, Spain). Serum albumin was determined using commercial kit (STANBIO Company, USA). Both of arginase and $\alpha$-L-fucosidase were measured using commercial kits (Bio-Diagnostic Company, Cairo, Egypt).

\section{Preparation of samples for Flow Cytometric analysis}

Liver tissues excised from rats were cut into pieces, and gently rubbed through fine nylon gauze (40-50 mesh count/cm, HD 140 Zuricher Buteltuch fabrik AG). Samples were then washed through the gauze with Tris/EDTA buffer of $\mathrm{pH}$ 7.5. Cells were suspended in PBS, centrifuged for 10 minutes at $1800 \mathrm{rpm}$, resuspended in sterile PBS. Prepared hepatic cells suspended in PBS at a concentration of 1 $\times 10^{6} \mathrm{cell} / \mathrm{mL}$.

\section{Proliferation marker Ki-67}

$\mathrm{Ki}-67$ is a cell-proliferation marker for tumor progression commonly used as an early predictor in cancer cells, its expression being associated with the prognosis of patients with HCC. HCC cells suspended in PBS at a concentration $1 \times 10^{6}$ cell $/ \mathrm{mL}$ were analyzed using mouse Ki-67 antibody protein (sc-7846 Per CP; Santa Cruz Biotechnology, Santa Cruz, $\mathrm{CA})$ according to the manufacturer's instructions.

\section{Cell apoptosis assay}

Cells $\left(1 \times 10^{6} /\right.$ well $)$ were washed with PBS, and resuspended in $300 \mu \mathrm{L}$ binding buffer. The cells were incubated with $5 \mu \mathrm{L}$ of fluorescein isothiocyanate-labeled annexin $\mathrm{V}$ stain for 15 minutes in the dark at room temperature. This was followed by the addition of $200 \mu \mathrm{L}$ of binding buffer. Further, $5 \mu \mathrm{L}$ of PI was added, and the cells were reincubated for 5 minutes at $37^{\circ} \mathrm{C}$. Apoptosis was quantized using a BD FACS Calibur flowcytometer, and data were analyzed by the BD CellQuest Pro (version 5.2) software.

\section{Cell cycle analysis}

A total of $1 \times 10^{6}$ cells were harvested, washed twice with cold PBS, fixed in $70 \%$ cold ethanol and stained with $5 \mu \mathrm{g} / \mathrm{ml}$ of PI in the dark for 15 min at $37^{\circ} \mathrm{C}$. The DNA content of the stained cells was detected using a BD FACS Calibur flowcytometer, and data were analyzed by the BD CellQuest Pro (version 5.2) software.

\section{95 Expression}

CD95 is a surface receptor that has the capacity to mediate apoptosis induction in cancer cells Expression of CD95 was determined by incubating aliquots of $1 \times 10^{6}$ cells in $100 \mu \mathrm{L}$ of wash buffer in the presence of respective $\mathrm{mAb}$ labeled with FITC conjugated mouse anti-human CD95 (Fas/Apo-1) mAb for 30 minutes at $4{ }^{\circ} \mathrm{C}$. After washing, the cells were fixed with $0.5 \%(\mathrm{w} / \mathrm{v})$ paraformaldehyde in D-PBS and flow cytometry analysis was performed.

\section{Statistical analysis}

Results are presented as mean \pm standard Errors (SEM).The statistical differences between groups were tested using a two-tailed Student's $t$ test. Statistical significance was assumed for $\mathrm{P}$ values $<0.05$. An IBM computer with a statistical software system MedCalc version 17.9.7 (MedCalc Software bvba, Ostend, Belgium) was used for these calculations.

\section{Results}

\section{Characterization of MSCs}

Under inverted microscope, undifferentiated MSCs were typical of adherent spindle and fibrocyte-like shape (Figure 1). Flow-cytometric analysis of cell surface markers in MSCs expressed CD29 and CD44 but did not express CD34 (data not shown). The surface marker expression pattern corresponds to bone marrow-MSCs. 

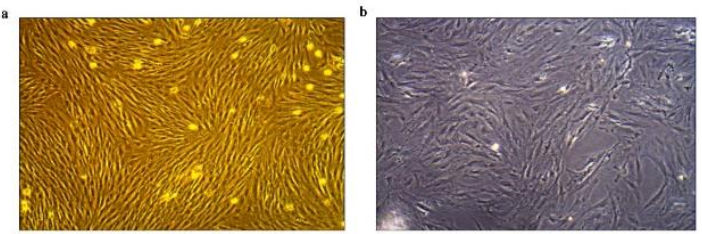

Figure 1 :(A): MSC culture after 14 days (magnification X 100). (B): MSCs at the $4^{\text {th }}$ passage (magnification X 100).

\section{Liver macroscopy and Histopathological analysis}

The macroscopic structure of livers was evaluated. The livers obtained from the HCC group were characterized by a flattened, fractured surface, and were of a darkred color, whereas those of the negative control group exhibited a smooth surface and a bright red color. All forms of treatments resulted in an improvement in the macroscopic condition of the liver as compared to 5FU-treated group (Figure 2).
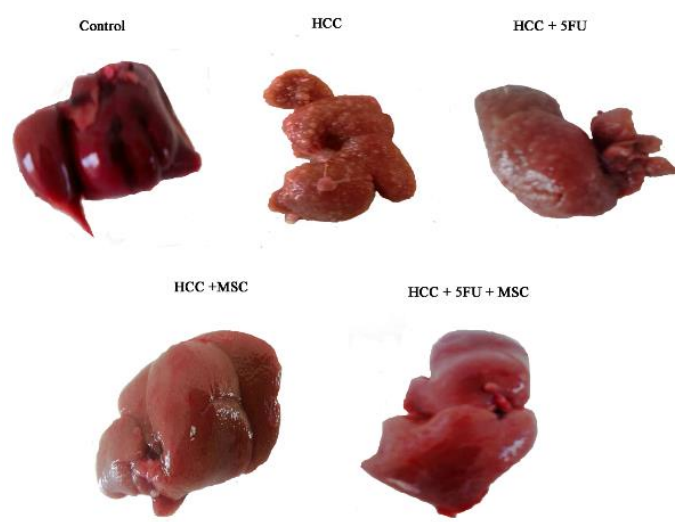

Figure 2: the morphological incidence of the liver of 5FU, MSCs, and their combination in DENinduced $\mathrm{HCC}$

In parallel, the histopathological changes of the liver structure (H\&E staining) was performed $\mathrm{H} \& \mathrm{E}$ staining of the sections from the control showed the typical cellular architecture, consisting of rounded single vesicular nuclei hepatocytes within the central vein, no portal inflammation, and regular lobular pattern. On the other hand, liver of HCC rat showed histopathological changes including marked liver fibrosis with cirrhotic changes and infiltration by mixed chronic inflammatory cells. Hepatocytes showed neoplastic changes in the form of more than two cell thick hepatic plates with sever degree dysplastic changes. On the other hand, Liver of HCC rat treated with $5 \mathrm{FU}$ is showing regression of inflammatory, fibrotic and dysplastic changes with remaining thick septa, while Liver of $\mathrm{HCC}$ rat treated with MSCs showed more regression of inflammatory, fibrotic and dysplastic changes with remaining thin septa and minimal inflammation, and liver section of HCC rat treated with 5FU plus MSCs showed marked improvement comparable to normal control group (Figure 3).

\section{Evaluation of liver function by serum biomarkers assessment}

ALT, AST, and albumin represent key enzymes for the evaluation of liver function, as they are released into the bloodstream from injured hepatocytes and their blood levels are increased under conditions of liver injury. In the current study, measurement of these enzymes was carried out using a spectrophotometer. ALT and AST activities were noticeably elevated in the HCC group while albumin level decreased compared to normal group.

Treating $\mathrm{HCC}$ rats with 5FU alone exhibited a significant elevation in ALT activity compared to normal rats but has no significant elevation with untreated HCC ( $p=<0.0001$ and $\mathrm{p}=0.08$, respectively). Moreover, ALT activity was significantly reduced in treated HCC group with MSCs compared to that untreated HCC and those treated with $5 \mathrm{FU}$ groups ( $p=0.03$ and $p<$ 0.001 , respectively). In addition, AST activity was significantly reduced in such group in comparison with untreated HCC $(p=0.002)$. Both ALT and AST activities were significantly reduced in MSCs plus 5FU treatment groups in comparison with both of untreated HCC and HCC treated with 5FU. Treating HCC rats with MSCs or MSCs together with 5FU exhibited increase in albumin levels compared to those treated with 5FU alone or with untreated HCC rats. It is noteworthy that the change in serum ALT, AST and albumin markers in MSCs plus 5FU treatment groups did not show a significant difference to the control group, indicating an approximate return of these enzymes to the normal state (Table 1).

\section{Serum tumor markers}

Tumor markers are potential screening tools that are widely used for early diagnosis of tumors. In this regard, Arginase and $\alpha-\mathrm{L}$ Fucosidase enzymes were evaluated. Table (1) showed that HCC group elicited dramatic 
significant increase $(\mathrm{p} \square 0.0001$ and $\mathrm{p}=0.0003$, respectively) in the tumor markers arginase and $\alpha$-L-fucosidase activities compared to untreated control rats. In cotrast, comparing to untreated HCC rats, HCC-5FU treated rats showed a significant reduction in arginase and $\alpha-\mathrm{L}-$

Table 1: Serum levels of cancer markers and liver damage enzymes

\begin{tabular}{lccccc}
\hline & $\begin{array}{c}\text { ALT } \\
(\mathbf{U} / \mathbf{L})\end{array}$ & $\begin{array}{c}\text { AST } \\
(\mathbf{U} / \mathbf{L})\end{array}$ & $\begin{array}{c}\text { Albumin } \\
(\mathbf{g} / \mathbf{d})\end{array}$ & $\begin{array}{c}\text { Arginase } \\
(\mathbf{U} / \mathbf{L})\end{array}$ & $\begin{array}{c}\boldsymbol{\alpha} \text {-L-Fucosidase } \\
(\mathbf{U} / \mathbf{L})\end{array}$ \\
\hline Untreated & $59.16 \pm 1.79$ & $119.5 \pm 6.9$ & $4.14 \pm 0.12$ & $25.16 \pm 1.74$ & $7.53 \pm 0.08$ \\
HCC & $158 \pm 3.9^{\mathrm{a}}$ & $315 \pm 4.64^{\mathrm{a}}$ & $2.28 \pm 0.15^{\mathrm{a}}$ & $88 \pm 5.04^{\mathrm{a}}$ & $10.9 \pm 0.26^{\mathrm{a}}$ \\
HCC+5FU & $223.16 \pm 6.6^{\mathrm{a}}$ & $157 \pm 10.1^{\mathrm{a}, \mathrm{b}}$ & $3.1 \pm 0.21^{\mathrm{a}, \mathrm{b}}$ & $43 \pm 5.82^{\mathrm{a}, \mathrm{b}}$ & $6.66 \pm 0.52^{\mathrm{b}}$ \\
HCC+MSCs & $74.5 \pm 7.41^{\mathrm{b}, \mathrm{c}}$ & $140.83 \pm 13.4^{\mathrm{b}}$ & $3.59 \pm 0.2^{\mathrm{a}, \mathrm{b}}$ & $34.16 \pm 2.6^{\mathrm{a}, \mathrm{b}}$ & $8.33 \pm 0.29^{\mathrm{b}, \mathrm{c}}$ \\
HCC+5FU+MSCs & $63.16 \pm 3.86^{\mathrm{b}, \mathrm{c}}$ & $124.33 \pm 5.85^{\mathrm{b}, \mathrm{c}}$ & $4.06 \pm 0.18^{\mathrm{b}, \mathrm{c}}$ & $27 \pm 2.7^{\mathrm{b}, \mathrm{c}}$ & $7.8 \pm 0.26^{\mathrm{b}}$ \\
\hline
\end{tabular}

Where HCC, Hepatocellular carcinoma; 5FU, 5-Fluorouracil; MSCs, Mesenchymal stem cells, a =significant when compared with the untreated, $b=$ significant when compared with the HCC, $\mathrm{c}=$ significant when compared with the 5FU.
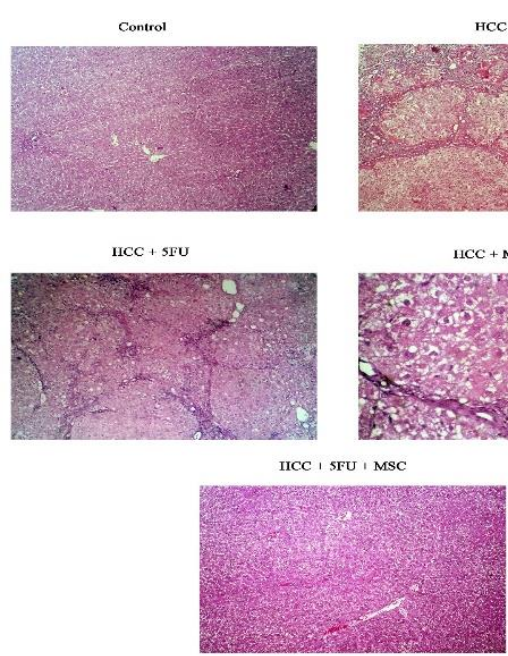

Figure 3: Liver histopathology. (A) Normal control rat liver showing normal liver structure $(\mathrm{H} \& \mathrm{E}, \mathrm{X}$ 100). (B) HCC control rat liver showing histopathological changes including marked liver fibrosis with cirrhotic changes and infiltration by mixed chronic inflammatory cells. Hepatocytes showed neoplastic changes in the form of more than two cell thick hepatic plates with sever degree dysplastic changes (H\&E, X 100). (C) Liver of HCC rat treated with $5 \mathrm{FU}$ is showing regression of inflammatory, fibrotic and dysplastic changes with remaining thick septa (H\&E, X 100). (D) Liver of HCC rat treated with MSCs showing more regression of inflammatory, fibrotic and dysplastic changes with remaining thin septa and minimal inflammation (H\&E, X 400). (E) Liver of HCC group treated with 5FU plus MSCs showed marked improvement comparable to normal control group (H\&E, X 100).

fucosidase activities $(\mathrm{p}=0.0002$ and $\mathrm{p}=0.002$, respectively), while rats in the $\mathrm{HCC}-\mathrm{MSC}$ group elicited a lower decreases in their activities $(\mathrm{p}=0.0001$ and $\mathrm{p}=0.002$, respectively). Furthermore, treated $\mathrm{HCC}$ with MSCs plus $5 \mathrm{FU}$ exhibited a significant down-regulation in arginase and $\alpha$-L-fucosidase activities compared to $\mathrm{HCC}$ rats. In addition, the latter group did not show a significant difference to the control group indicating an approximate return to the normal state.

\section{KI 67}

Untreated HCC rats exhibited a significant elevation in KI 67 expression compared to control $(p=0.001)$. In addition, 5FU treatment induces a significant elevation in KI 67 expression compared to control ( $p=0.0014)$. However, treated HCC rats with MSCs exhibited a significant reduction in such expression compared to control, HCC and treated HCC rats with 5FU ( $p=0.01, p=0.001$ and $p=0.001$, respectively). Moreover, the HCC treated with combination of MSCs and 5FU resulted in a significant reduction in KI 67 expression when compared to both of HCC and treated $\mathrm{HCC}$ rats with 5FU ( $p=0.002$ and $p=$ 0.001 , respectively). On the other hand, there was a significant elevation between the latter group and control $(p=0.02)$ and no significant change with HCC treated with MSCs (Figure 4).

\section{Annexin}

Cellular status was defined as follows: unstained cells were classified as 'alive'; cells stained only by Annexin $\mathrm{V}$ were classified as 'early apoptotic'; cells stained by both Annexin $\mathrm{V}$ and PI were classified as 'late apoptotic'; and cells stained only by PI were classified as 'necrotic'. An insignificant increase in the percentage of early apoptotic cells has been observed in untreated HCC group. On the other hand, the latter group resulted in a significant elevation in the percentage of late apoptotic and necrotic cells $(p=0.03$ and $p=0.02$, respectively). 

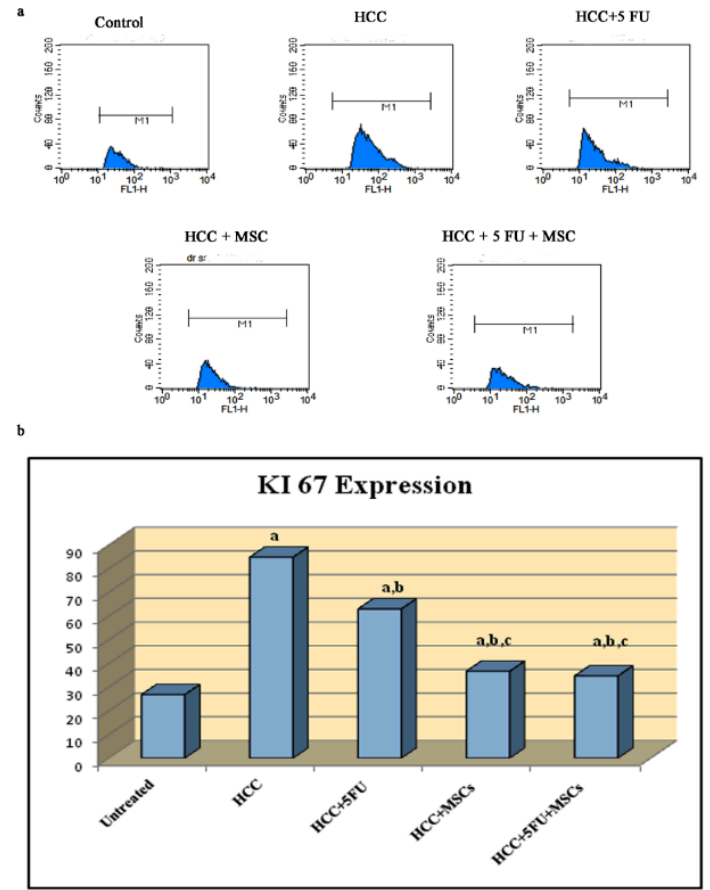

Figure 4: Effect of treatment with MSCs, 5FU and their combination on the KI67 expression (a) Flowcytometric quantification of KI67 expression on HCC- rats (b) Bar chart representing KI67 expression in different treatments groups. Significant alterations are expressed relative to untreated rats (marked with asterisks ${ }^{\text {a)}}$ ), to HCC (marked with crosses b), to 5FU (marked with crosses c), and to MSCs (marked with ampersand d). Statistical difference was considered significant $p<$ 0.05 .

Treated HCC rats with 5FU resulted in a significant elevation in the percentage of cells at both of the late apoptotic and the necrotic phases ( $p=0.004$ and $p=0.009$, respectively) compared to normal group. However, the same group exhibited an insignificant reduction in early apoptosis compared with the untreated control and HCC groups. Furthermore, HCC group treated with MSCs showed a significant reduction in the percentage of late apoptotic cells compared to untreated control, HCC and treated HCC with 5FU groups $(p=0.005, p=$ 0.004 and $p=0.0008$, respectively). In contrast, an insignificant decrease in the percentage of early apoptotic cells has been found in the latter treatment when compared to all experimental groups. Except normal group, such treatment had a significant reduction in the percentage of necrotic cells when compared to either those treated with $5 \mathrm{FU}$ or untreated HCC ( $p=0.01$ and $p=0.002$, respectively).

Interestingly, HCC rats treated with a combination of MSCs and 5FU showed an insignificant elevation in the percentage of early apoptotic cells compared to all treated groups. Also, treatment of rats with such combination resulted in a significant change in the percentage of cells which were at the late apoptotic phase compared to those treated with MSCs and 5FU $(p=0.01$ and $p=0.003$, respectively, Figure 5). On the contrary, an insignificant elevation in late apoptotic cells as compared to those untreated control and HCC rats was observed in such treatment.

\section{MSC influences the cell cycle}

Cell cycle arrest was analyzed by PI quantification using flow cytometry. Compared to control, there was an increase in the cell population at $\mathrm{S}$ and $\mathrm{G} 2 / \mathrm{M}$ phases in HCC group, while the $\mathrm{G}_{0}-\mathrm{G}_{1}$ phase showed a decrease in the percentage of cells. Treating $\mathrm{HCC}$ with $5 \mathrm{FU}$ exhibited a reduction in percentage of cells in $\mathrm{G}_{0}-\mathrm{G}_{1}$ phase and a significant increase in the cell population at $\mathrm{S}$ and $\mathrm{G} 2 / \mathrm{M}$ phases $(p=0.001$ and $p=0.02$, respectively) as compared to control group. Compared to both untreated $\mathrm{HCC}$ and treated $\mathrm{HCC}$ with 5FU, there was a subsequent elevation in percentage of cells in $\mathrm{G}_{0}-\mathrm{G}_{1}$

phase at HCC treated with MSCs, while the S, G2/M-phases showed consistent decline in percentage of cells. Essentially, MSCs plus 5FU treated $\mathrm{HCC}$ rats showed $\mathrm{G}_{0}-\mathrm{G}_{1}$ arrest with a significant decrease in the cell population at $\mathrm{S}$ and $\mathrm{G} 2 / \mathrm{M}$ phases (Figure 6).

\section{CD95 expression}

Untreated HCC rats exhibited a significant elevation in CD95 expression compared to control $(p=0.0001)$. HCC rats treated with 5FU induces a significant elevation in such marker compared to control group but showed a significant reduction compared to HCC group ( $p=0.03$ and $p=0.002$, respectively). However, treated HCC rats with MSCs exhibited a significant reduction in CD95 expression when compared to both of $\mathrm{HCC}$ and treated $\mathrm{HCC}$ rats with 5FU $(p=0.0001$ and $p=$ 0.0004 , respectively). Furthermore, HCC treated with MSCs plus 5FU resulted in a significant reduction in CD95 expression when compared to both of HCC and treated HCC rats with 5FU ( $p=0.0001)$. On the other hand, there was no significant change between the latter group and both of control and treated HCC with MSCs (Figure 7). 


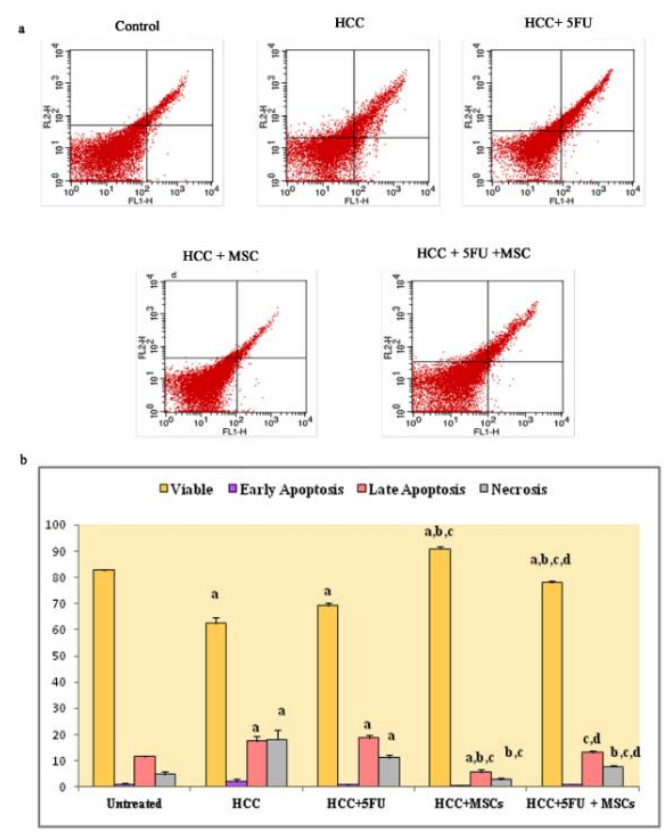

Figure 5: Effect of treatment with MSCs, 5FU and their combination on the induction of apoptosis (a) Flowcytometric quantification of apoptosis in different groups after staining with Annexin V and PI (b) Bar chart representing early , late apoptosis and necrosis. Significant alterations are expressed relative to untreated rats (marked with asterisks ${ }^{\text {a }}$ ), to HCC (marked with crosses b), to 5FU (marked with crosses c), and to MSCs (marked with ampersand d). Statistical difference was considered significant $p<$ 0.05 .
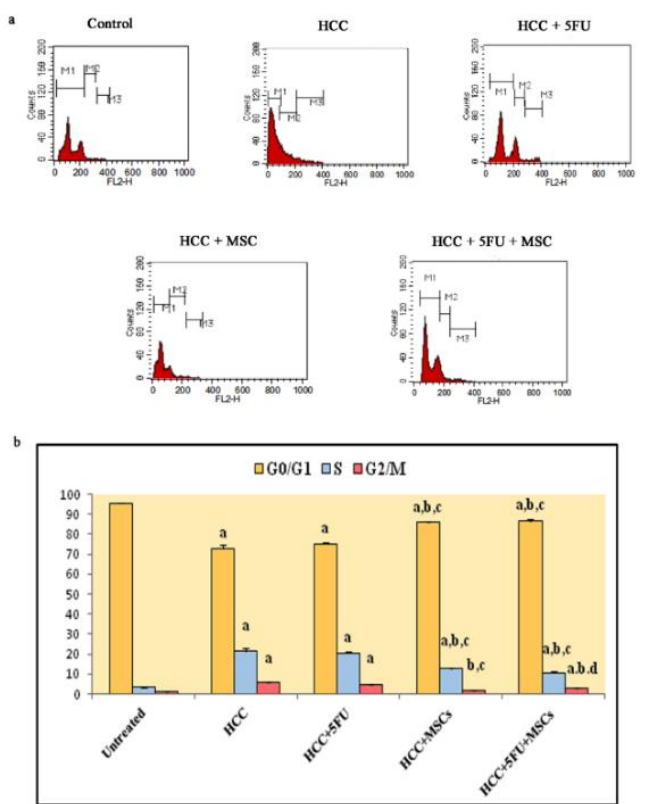

Figure 6: (a) Histographs of cell cycle distribution of untreated control rats and HCC- treated rats. (b) Bar graphs showing the percentage of cells in G0/G1, S, and G2/M phases. Significant alterations are expressed relative to untreated rats (marked with asterisks ${ }^{a}$ ), to $\mathrm{HCC}$ (marked with crosses b), to 5FU (marked with crosses c), and to MSCs (marked with ampersand d). Statistical difference was considered significant $p<0.05$.
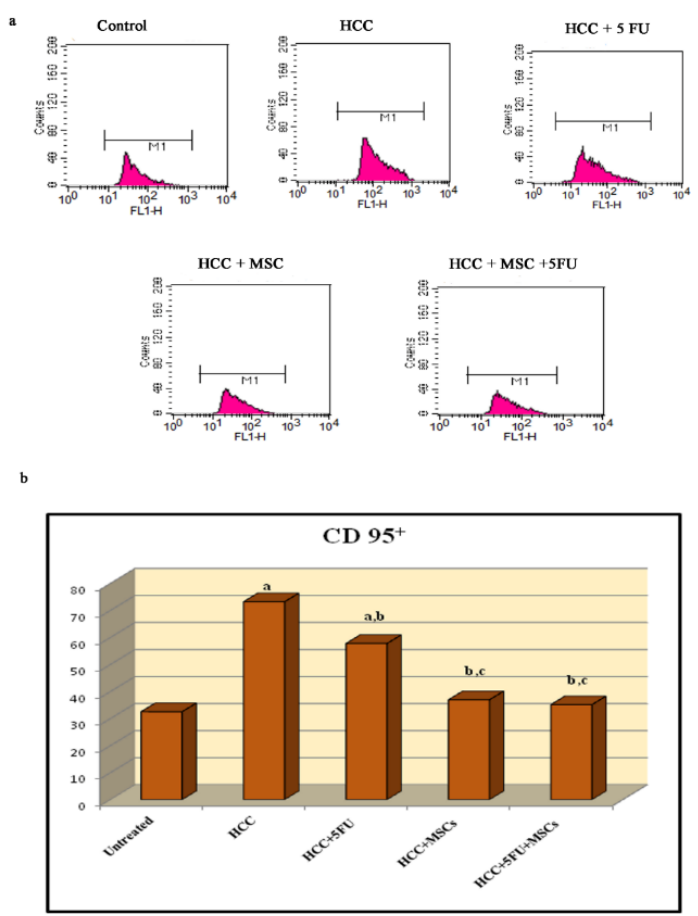

Figure 7: Effect of treatment with MSCs, 5FU and their combination on the CD95 expression (a) Flowcytometric quantification of CD95 expression on HCC- rats (b) Bar chart representing CD95 expression in different treatments groups. Significant alterations are expressed relative to untreated rats (marked with asterisks ${ }^{a}$ ), to $\mathrm{HCC}$ (marked with crosses b), to 5FU (marked with crosses c), and to MSCs (marked with ampersand d). Statistical difference was considered significant $p<$ 0.05 .

\section{Discussion}

HCC is a highly aggressive tumor standing the third foremost cause of cancer death in the world (Ferlay, Colombet et al. 2019). Even though surgery, novel chemotherapies and radiotherapies are always improving, the prognosis of $\mathrm{HCC}$ remains awfully deprived. Consequently, elucidating the vital proceedings underlying HCC tumorigenesis and exploring new therapeutic strategies to control the tumor progression is the essential matter to prolong patient survival (Chen, Ji et al. 2019). 5-FU-based chemotherapy was widely used for the treatment of HCC. However, the drug resistance and high toxicity limited the efficacy of 5-FU (Gao, Yu et al. 2018). Therefore, it is necessary to find more effective chemotherapeutic drugs and/or combination therapy for $\mathrm{HCC}(\mathrm{Xu}, \mathrm{Guo}$ et al. 2020). MSCs are a group of adult stem 
cells which can migrate to tissue injury sites and tumor sites. Due to their capability to migrate as well as differentiate, MSCs play a pivotal role in the regeneration of connective tissues, preservation of tissue integrity and wound repairment (Chen, Ji et al. 2019). MSCs can represent a hope for cancer therapies through their potential of inhibiting tumor cells from regeneration in different types of animal model. In this study, we are going to compare the antitumor properties of 5FU plus MSCs combination therapy with using either of the agents alone.

In our study, to evaluate the effect of MSCs on HCC, rats treated with $\mathrm{N}$-diethylnitrosamine (DEN) as rat liver cancer model that resembles human primary HCC. Hepatic damage caused by DEN obviously represents the dysfunction of the metabolism of liver cells, resulting in distinctive changes in the serum enzymes levels. In this regard, DEN-induced hepatic disruption, subsequent leakage of cytoplasmic and membrane-bound enzymes from the neoplastic cells into circulation resulted in elevation of serum ALT, AST activities and reduction in albumin levels (Singh, Singh et al. 2018). Besides, Ikeda et al., who reported that the inhibition of albumin was a marker in hepatocarcinogensis (Ikeda, Arase et al. 2000). Moreover, Evaluation of liver function showed that activity of ALT was markedly increased in response to treatment with 5-FU compared to $\mathrm{HCC}$ rats. Also, treating $\mathrm{HCC}$ rats with $5 \mathrm{FU}$ showed a significant decrease in AST activity with the simultaneous elevation in albumin level as regard to HCC rats but still higher than normal rats. These results may confirm those of Dimitriu, and Gelen, who demonstrated that 5FU resulted in elevation in serum ALT and AST activities due to severe hepatotoxicity (Dimitriu, LUPUSORU et al. 2015, Gelen, Şengül et al. 2018). On the other hand, the significant decrease of serum ALT and AST as well as significant increase of albumin in both HCC groups treated with either MSCs alone or MSCs plus 5FU suggested improvement of liver function. No significant difference was found in the liver enzyme levels of both treated HCC groups with MSCs either alone or in combination with $5 \mathrm{FU}$ when compared with a healthy control group, which indicated normalization of liver functions (Abdelhady, El-Abasy et al. 2017, El-Magd, Mohamed et al. 2019). The previous results as well as the histopathological picture that showed minimal reversible liver cell damage and absence of fibrous thickening of portal tracts and inflammation which drew attention to the potential of MSCs in regenerative therapy (Abdel aziz, El Asmar et al. 2011).

Importantly, Tumor markers arginase and $\alpha-L$-Fucosidase were significantly increased in rats treated with DEN compared to normal rats that indicating tumor cell proliferation (Hussein and Khalifa 2014). Due to hepatocyte damage, arginase leak rapidly from hepatocytes that expressed predominantly in the liver which catalyzes the conversion of arginine to ornithine and urea, completing the last step in the urea cycle (Merrick, Bruno et al. 2006).The previous result is in accordance with Satriano, and Cederbaum et al., who demonstrated that increased stimulation of arginase expression leads to production of polyamines that promote tumor cell proliferation (SATRIANO 2003, Hussein and Khalifa 2014). Treated rats with DEN enhanced the higher expression of $\alpha$ - L -Fucosidase which may confirm those of Sivaramakrishnan et al., who reported that the serum $\alpha-\mathrm{L}$ fucosidase activity level elevated in HCC (Sivaramakrishnan, Shilpa et al. 2008). Moreover, treating $\mathrm{HCC}$ rats with $5 \mathrm{FU}$ exhibited a reduction in both tumor markers levels by half than HCC. It is worth mentioning that the increase in arginase caused innate error in urea cycle was the main factor that caused increased hepatocyte damage due to the high liver toxicity (Ragaei, Mansy et al. 2016). Treatment HCC with MSCs alone, both of arginase and $\alpha-\mathrm{L}$-Fucosidase enzyme were decreased significantly than untreated HCC rats. Notably, the co-supplementation of MSCs with 5FU to $\mathrm{HCC}$ rats exhibited no significant differences in such enzyme levels as regard to normal rats which showed acceptable outcomes when their tumor marker levels were low. The previous results may confirm that MSCs have antitumor properties.

To determine whether the observed tumor growth suppression was caused by inhibition of cell proliferation, KI67 was used as a marker of cell proliferation. treatment of rats with DEN revealed an elevation in KI67 which significantly associated with advanced stage of HCC, such explanation has been reported by (Luo, Ren et al. 2015). Treatment of $\mathrm{HCC}$ rats with $5 \mathrm{FU}$ exhibited a significant elevation in KI67 expression compared to normal rats and in respect to $\mathrm{HCC}$ rats they 
showed a significant down-regulation in such marker. The previous result revealed that 5-FU reduce $\mathrm{HCC}$ cell proliferation and these observation in agreement with Liu et al., who repoted that 5FU inhibited $\mathrm{HCC}$ cell growth (Liu, Wu et al. 2018). Furthermore, treatment of rats with the combination therapy of $5 \mathrm{FU}$ together with MSCs and MSCs alone significantly reduced the proliferation index of HCC tumors compared with 5FU alone that may improve the anticancer effect of 5FU. This study assumes that MSCs may inhibit the progression of hepatic carcinogenesis particularly by inhibiting cell proliferation. These results may confirm those of Zong et al. who demonstrated that MSCs exert an antiproliferative effect and could protect hepatocytes from DEN damage by inhibiting cell death and finally had a tumor-suppressive effect (Yulyana, Ho et al. 2015, Zong, Zhang et al. 2018).

Treating rats with DEN resulted in a significant increase in cell populations in late apoptotic and necrotic phases, which mediate cell death. Previous reports suggest that DEN administration resulted in DNA damage and ROS production in liver cells, which could contribute to cell apoptosis (Zong, Zhang et al. 2018). Therefore, to ascertain whether the antiproliferative effect of MSC is through initiation of programmed cell death or necrosis, $\mathrm{HCC}$ rats treated with MSCs alone and in combination with 5FU were tested for Annexin $\mathrm{V}$ expression analysis. However, the inhibition is neither due to apoptosis nor necrosis. Although MSCs did not increase the percentage of early apoptotic cell population, a slight reduction of apoptosis is notable in the presence of MSC. This demonstrates that MSCs induced inhibition is not due to a killing process, inflicting a direct control on cell cycle machinery of tumour cells so it might be due to a pathway other than apoptosis. The previous observation may be in accordance with Sarmadi et al., who reported similar explanation (Sarmadi, Tong et al. 2010).

Notably, treating HCC with MSCs alone or in combination with 5FU predominantly arrest the cell cycle progression of tumor cells in the G0/G1 phase of the cell cycle, and thus fewer cells proceeded to the subsequent $\mathrm{S}$ and $\mathrm{G} 2 / \mathrm{M}$ phases. The observed disruption of cell cycle progression in the tumor cells suggests that treatment with MSCs leads to potent growth inhibition of tumor cells without being subject to apoptosis (Sarmadi, Ahmadloo et al. 2020).

It is worth mentioning that CD95, a cell surface protein that belongs to the tumor necrosis factor receptor family, can mediate apoptosis. Also, CD95 is required for efficient liver regeneration following partial hepatectomy (Chen, Park et al. 2011). Treating rats with DEN showed a significant elevation in CD 95 expression that indicates tumor proliferation. These observations are in accordance with the previous study reported by Reinehr et al, (Reinehr, Sommerfeld et al. 2008). Treating HCC rats with 5FU alone exhibited an elevation in such marker compared to normal rats with a reduced expression regard to HCC group stimulating CD95 on HCC cells that may be due to the resistance of cells to $5 \mathrm{FU}$ therapy (Peter, Hadji et al. 2015). On the other hand, treating HCC rats with MSCs or MSCs plus 5FU revealed that no significant difference in CD 95 expression compared to normal control. The latter treatment resulted in a significant reduction in that marker compared to those of HCC treated with 5FU alone or untreated HCC. These observations suggesting that MSCs might decrease the susceptibility to apoptotic signals. Besides, the correlation of the change in the parameters of the cell cycle distribution with the Annexin $\mathrm{V}$ results via MSCs treatment may confirm the previous findings.

In conclusion, this study revealed that MSCs enhanced the efficacy of chemotherapy in $\mathrm{HCC}$ rats alone or in combination with 5FU reducing its side effects via improvement of liver enzymes, reduction of tumor markers; decrease the tumor cells proliferation, cell cycle arrest. Therefore, the cells continued to retain their characteristics.

\section{Conflicts of interests}

The authors declare that they have no conflicts of interests.

\section{Compliance with Ethical Standards}

All procedures performed in in the study followed the relevant ethical standards of the institutional or national research committee (Ethics Board of Helwan University with the 1964 Helsinki declaration and its later 
amendments or comparable ethical standards.

\section{References}

Abdel aziz, M. T., M. F. El Asmar, H. M. Atta, S. Mahfouz, H. H. Fouad, N. K. Roshdy, L. A. Rashed, D. Sabry, A. A. Hassouna and F. M. Taha (2011). "Efficacy of mesenchymal stem cells in suppression of hepatocarcinorigenesis in rats: possible role of Wnt signaling." Journal of Experimental \& Clinical Cancer Research 30(1): 49.

Abdelhady, D., M. El-Abasy, S. Abou-Asa, Z. Elbialy, M. Shukry, A. Hussein, A. Saleh and M. El-Magd (2017). "The ameliorative effect of Aspergillus awamori on aflatoxin B1-induced hepatic damage in rabbits." World Mycotoxin Journal 10(4): 363-373.

Babajani, A., P. Soltani, E. Jamshidi, M. H. Farjoo and H. Niknejad (2020). "Recent Advances on Drug-Loaded Mesenchymal Stem Cells With Anti-neoplastic Agents for Targeted Treatment of Cancer." Frontiers in Bioengineering and Biotechnology 8.

Calabrò, L., E. Fonsatti, G. Bellomo, A. Alonci, F. Colizzi, L. Sigalotti, M. Altomonte, C. Musolino and M. Maio (2003). "Differential levels of soluble endoglin (CD105) in myeloid malignancies." Journal of cellular physiology 194(2): 171-175.

Chen, J., T. Ji, D. Wu, S. Jiang, J. Zhao, H. Lin and X. Cai (2019). "Human mesenchymal stem cells promote tumor growth via MAPK pathway and metastasis by epithelial mesenchymal transition and integrin $\alpha 5$ in hepatocellular carcinoma." Cell death \& disease 10(6): 1-12.

Chen, L., S.-M. Park, A. V. Tumanov, A. Hau, K. Sawada, C. Feig, J. R. Turner, Y.-X. Fu, I. L. Romero and E. Lengyel (2011). "CD95 promotes tumour growth." Natur 471(7337): 254.

Council, N. R. (2010). Guide for the care and use of laboratory animals, National Academies Press.

Daher, S., M. Massarwa, A. A. Benson and T. Khoury (2018). "Current and future treatment of hepatocellular carcinoma: an updated comprehensive review." Journal of clinical and translational hepatology 6(1): 69.

Damdinsuren, B., H. Nagano and M. Monden (2007). "Combined intra-arterial 5-fluorouracil and subcutaneous interferon-alpha therapy for highly advanced hepatocellular carcinoma." Hepatology Research 37: S238-S250.

Dimitriu, D.-C., C.-E. LUPUSORU, I. Cojocaru, C. Gafitanu, L. Palade and R.-V. Lupusoru (2015). "ASSESSING BIOCHEMICAL AND OXIDATIVE STRESS PARAMETERS AFTER VAGINAL AND ORAL ADMINISTRATION
OF 5-FLUOROURACIL IN LABORATORY

ANIMALS." Farmacia 63(2): 230-233.

El-Magd, M. A., Y. Mohamed, E. S. El-Shetry, S. A. Elsayed, M. A. Gazia, G. A. Abdel-Aleem, N. M. Shafik, W. S. Abdo, N. I. El-Desouki and M. A. Basyony (2019). "Melatonin maximizes the therapeutic potential of non-preconditioned mscs in a den-induced rat model of hcc." Biomedicine \& Pharmacotherapy 114: 108732.

Ferlay, J., M. Colombet, I. Soerjomataram, C. Mathers, D. Parkin, M. Piñeros, A. Znaor and F. Bray (2019). "Estimating the global cancer incidence and mortality in 2018: GLOBOCAN sources and methods." International journal of cancer 144(8): 1941-1953.

Gao, F., X. Yu, R. Meng, J. Wang and L. Jia (2018). "STARD13 is positively correlated with good prognosis and enhances 5-FU sensitivity via suppressing cancer stemness in hepatocellular carcinoma cells." OncoTargets and therapy 11: 5371.

Garg, M., L. Lincz, K. Adler, F. Scorgie, S. Ackland and J. Sakoff (2012). "Predicting 5-fluorouracil toxicity in colorectal cancer patients from peripheral blood cell telomere length: a multivariate analysis." British journal of cancer 107(9): 1525-1533.

Gelen, V., E. Şengül, S. Yıldırım and G. Atila (2018). "The protective effects of naringin against 5-fluorouracil-induced hepatotoxicity and nephrotoxicity in rats." Iranian journal of basic medical sciences 21(4): 404.

Godwin, E., N. Young, J. Dudhia, I. Beamish and R. Smith (2012). "Implantation of bone marrowderived mesenchymal stem cells demonstrates improved outcome in horses with overstrain injury of the superficial digital flexor tendon." Equine veterinary journal 44(1): 25-32.

Greten, T. F. (2020). "Treatment of Liver Cancer." The Liver: Biology and Pathobiology: 782-791.

Hemming, A. W., J. Berumen and K. Mekeel (2016). "Hepatitis B and hepatocellular carcinoma." Clinics in liver disease 20(4): 703-720.

Hernanda, P. Y., A. Pedroza-Gonzalez, L. J. van der Laan, M. E. Bröker, M. J. Hoogduijn, J. N. Ijzermans, M. J. Bruno, H. L. Janssen, M. P. Peppelenbosch and Q. Pan (2013). "Tumor promotion through the mesenchymal stem cell compartment in human hepatocellular carcinoma." Carcinogenesis 34(10): 2330-2340.

Hu, Z., G. Lv, Y. Li, E. Li, H. Li, Q. Zhou, B. Yang and W. Cao (2016). "Enhancement of anti-tumor effects of 5-fluorouracil on hepatocellular carcinoma by low-intensity ultrasound." Journal of Experimental \& Clinical Cancer Research 35(1): 1-12.

Hussein, R. H. and F. K. Khalifa (2014). "The 
protective role of ellagitannins flavonoids pretreatment against N-nitrosodiethylamine induced-hepatocellular carcinoma." Saudi Journal of Biological Sciences 21(6): 589-596.

Ikeda, K., Y. Arase, S. Saitoh, M. Kobayashi, Y. Suzuki, F. Suzuki, A. Tsubota, K. Chayama, N. Murashima and H. Kumada (2000). "Interferon beta prevents recurrence of hepatocellular carcinoma after complete resection or ablation of the primary tumor - a prospective randomized study of hepatitis C virus-related liver cancer." Hepatology 32(2): 228-232.

Karimov, K. Y., F. Inoyatova and M. Mukhamedova (2003). "Changes in some indices of the synthesis of nitric oxide during the early stages of hepatocarcinogenesis." Experimental and Toxicologic Pathology 55(1): 17-19.

Li, J.-Y., K.-K. Ren, W.-J. Zhang, L. Xiao, H.-Y. Wu, Q.-Y. Liu, T. Ding, X.-C. Zhang, W.-J. Nie and Y. Ke (2019). "Human amniotic mesenchymal stem cells and their paracrine factors promote wound healing by inhibiting heat stress-induced skin cell apoptosis and enhancing their proliferation through activating PI3K/AKT signaling pathway." Stem cell research \& therapy 10(1): 1-17.

Liu, C., Y. Zhao, J. Wang, Y. Yang, Y. Zhang, X. Qu, S. Peng, Z. Yao, S. Zhao and B. He (2020). "FoxO3 reverses 5-fluorouracil resistance in human colorectal cancer cells by inhibiting the Nrf2/TR1 signaling pathway." Cancer Letters 470: 29-42.

Liu, Q. W., J. Y. Li, X. C. Zhang, Y. Liu, Q. Y. Liu, L. Xiao, W. J. Zhang, H. Y. Wu, K. Y. Deng and H. B. Xin (2020). "Human amniotic mesenchymal stem cells inhibit hepatocellular carcinoma in tumour-bearing mice." Journal of Cellular and Molecular Medicine 24(18): 1052510541

Liu, T., X. Wu, Y. Li, W. Lu, F. Zheng, C. Zhang, Q. Long, H. Qiu, Y. Li and Q. Ge (2018). "RBFOX3 regulates the chemosensitivity of cancer cells to 5-fluorouracil via the PI3K/AKT, EMT and cytochrome-C/caspase pathways." Cellular Physiology and Biochemistry 46(4): 1365-1380.

Luo, Y., F. Ren, Y. Liu, Z. Shi, Z. Tan, H. Xiong, Y. Dang and G. Chen (2015). "Clinicopathological and prognostic significance of high Ki-67 labeling index in hepatocellular carcinoma patients: a meta-analysis." International journal of clinical and experimental medicine 8(7): 10235 .

Merrick, B. A., M. E. Bruno, J. H. Madenspacher, B. A. Wetmore, J. Foley, R. Pieper, M. Zhao, A. J. Makusky, A. M. McGrath and J. X. Zhou (2006). "Alterations in the rat serum proteome during liver injury from acetaminophen exposure."
Journal of Pharmacology and Experimental Therapeutics 318(2): 792-802.

Moris, D., L. Lu and S. Qian (2017). "Mechanisms of liver-induced tolerance." Current opinion in organ transplantation 22(1): 71-78.

Peter, M., A. Hadji, A. Murmann, S. Brockway, W. Putzbach, A. Pattanayak and P. Ceppi (2015). "The role of CD95 and CD95 ligand in cancer." Cell death \& differentiation 22(4): 549-559.

Qin, S., Y. Bai, H. Y. Lim, S. Thongprasert, Y. Chao, J. Fan, T.-S. Yang, V. Bhudhisawasdi, W. K. Kang and Y. Zhou (2013). "Randomized, multicenter, open-label study of oxaliplatin plus fluorouracil/leucovorin versus doxorubicin as palliative chemotherapy in patients with advanced hepatocellular carcinoma from Asia." J Clin Oncol 31(28): 3501-3508.

Ragaei, A., A. Mansy and D. Sabry (2016). "Therapeutic Potential of Mesenchymal Stem Cells and Vitamin E on Experimental Hepatocellular Carcinoma." J Stem Cell Res Ther 6(362): 2.

Reinehr, R., A. Sommerfeld and D. Häussinger (2008). "CD95 ligand is a proliferative and antiapoptotic signal in quiescent hepatic stellate cells." Gastroenterology 134(5): 1494-1506. e1497.

Sarmadi, V., C. Tong, S. Vidyadaran, M. Abdullah, H. Seow and R. Ramasamy (2010). "Mesenchymal stem cells inhibit proliferation of lymphoid origin haematopoietic tumour cells by inducing cell cycle arrest." Med J Malaysia 65(3): 209-214.

Sarmadi, V. H., S. Ahmadloo, M. H. Boroojerdi, C. M. John, S. J. R. al-Graitte, H. Lawal, M. Maqbool, L. K. Hwa and R. Ramasamy (2020). "Human Mesenchymal Stem Cells-mediated Transcriptomic Regulation of Leukemic Cells in Delivering Anti-tumorigenic Effects." Cell Transplantation 29: 0963689719885077.

SATRIANO, J. (2003). "Agmatine: at the crossroads of the arginine pathways." Annals of the New York Academy of Sciences 1009(1): 34-43.

Serhal, R., N. Saliba, G. Hilal, M. Moussa, G. S. Hassan, O. El Atat and N. Alaaeddine (2019). "Effect of adipose-derived mesenchymal stem cells on hepatocellular carcinoma: In vitro inhibition of carcinogenesis." World journal of gastroenterology 25(5): 567.

Sho, T., M. Nakanishi, K. Morikawa, M. Ohara, N. Kawagishi, T. Izumi, M. Umemura, J. Ito, M. Nakai and G. Suda (2017). "A phase I study of combination therapy with sorafenib and 5fluorouracil in patients with advanced hepatocellular carcinoma." Drugs in R\&D 17(3): 381-388.

Singh, D., M. Singh, E. Yadav, N. Falls, D. S. Dangi, V. Kumar, P. W. Ramteke and A. Verma (2018). 
"Attenuation of diethylnitrosamine (DEN)Induced hepatic cancer in experimental model of Wistar rats by Carissa carandas embedded silver nanoparticles." Biomedicine \& Pharmacotherapy 108: 757-765.

Sivaramakrishnan, V., P. N. M. Shilpa, V. R. P. Kumar and S. N. Devaraj (2008). "Attenuation of $\mathrm{N}$-nitrosodiethylamine-induced hepatocellular carcinogenesis by a novel flavonol-Morin." Chemico-biological interactions 171(1): 79-88.

Stoltz, A., J. Gagnière, A. Dupré and M. Rivoire (2014). "Radiofrequency ablation for colorectal liver metastases." Journal of Visceral Surgery 151: S33-S44.

Winocur, G., J. Vardy, M. A. Binns, L. Kerr and I. Tannock (2006). "The effects of the anti-cancer drugs, methotrexate and 5-fluorouracil, on cognitive function in mice." Pharmacology Biochemistry and Behavior 85(1): 66-75.

Wu, N., Y.-L. Zhang, H.-T. Wang, D.-W. Li, H.-J. Dai, Q.-Q. Zhang, J. Zhang, Y. Ma, Q. Xia and J.-M. Bian (2016). "Overexpression of hepatocyte nuclear factor $4 \alpha$ in human mesenchymal stem cells suppresses hepatocellular carcinoma development through
Wnt/ $\beta$-catenin signaling pathway downregulation." Cancer biology \& therapy 17(5): 558-565.

Xu, T., P. Guo, C. Pi, Y. He, H. Yang, Y. Hou, X. Feng, Q. Jiang, Y. Wei and L. Zhao (2020). "Synergistic Effects of Curcumin and 5Fluorouracil on the Hepatocellular Carcinoma In vivo and vitro through regulating the expression of COX-2 and NF-кB." Journal of Cancer 11(13): 3955.

Yulyana, Y., I. A. Ho, K. C. Sia, J. P. Newman, X. Y. Toh, B. B. Endaya, J. K. Chan, M. Gnecchi, H. Huynh and A. Y. Chung (2015). "Paracrine factors of human fetal MSCs inhibit liver cancer growth through reduced activation of IGF1R/PI3K/Akt signaling." Molecular Therapy 23(4): 746-756.

Zong, C., H. Zhang, X. Yang, L. Gao, J. Hou, F. Ye, J. Jiang, Y. Yang, R. Li and Z. Han (2018). "The distinct roles of mesenchymal stem cells in the initial and progressive stage of hepatocarcinoma." Cell death \& disease 9(3): 114.

\section{عنوان البحث: الدور العلاجى المحتمل للخلايا الجدعية من النسيج المتوسط لنمودج سرطان الكبد فى الجردان المان}

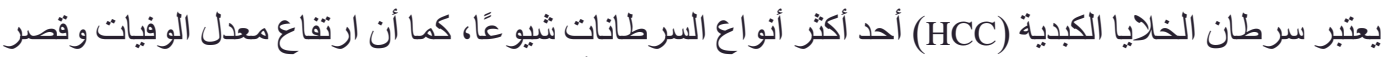

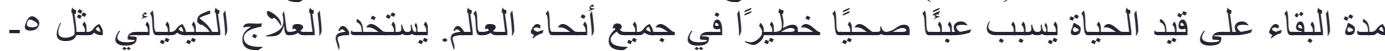

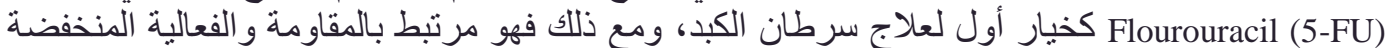

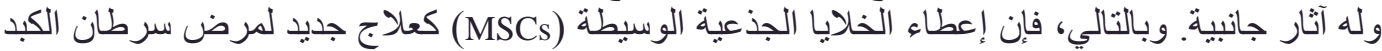

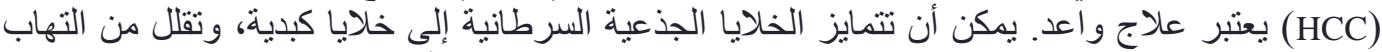

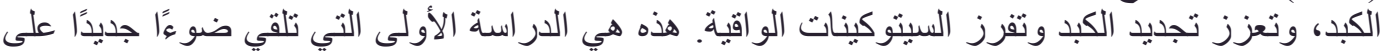

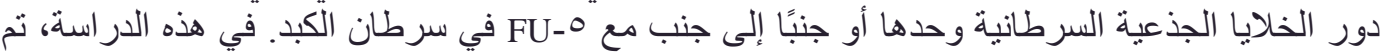

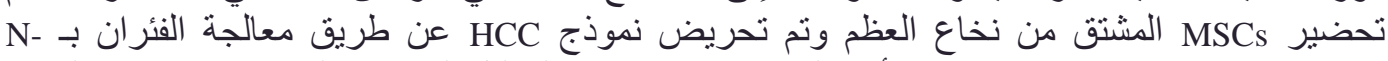
diethylnitrosamine (DEN)

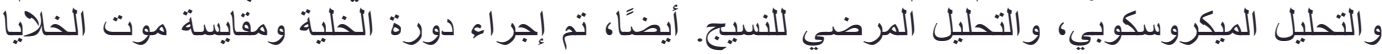

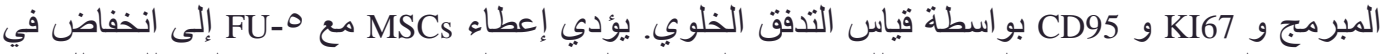

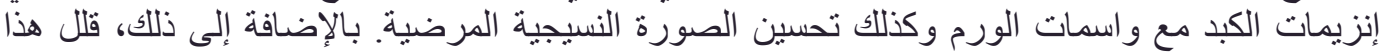

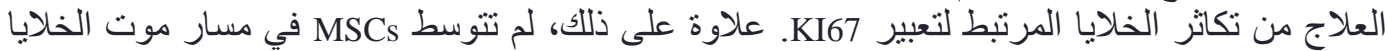

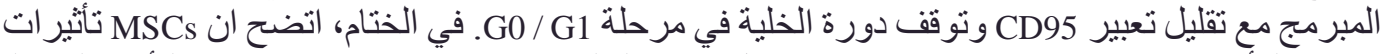

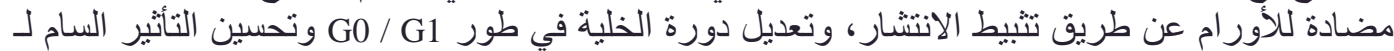

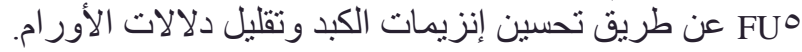

